\title{
Exploitation of Adaptations of Animal Parasites - A Key towards Designing Effective Control Strategies against Parasitic Diseases of Animals
}

\author{
Siju Susan Jacob ${ }^{1 *}$, P.P. Sengupta ${ }^{1}$, J.K. Chamuah ${ }^{2}$, O.K. Raina ${ }^{3}$, \\ M. Sankar ${ }^{4}$, T.G. Sumithra ${ }^{5}$ and A. Prajapati ${ }^{1}$ \\ ${ }^{1}$ ICAR-NIVEDI, Bangalore, Karnataka, India \\ ${ }^{2}$ ICAR-NRC on Mithun, Nagaland, India \\ ${ }^{3} C A R$ - Indian Veterinary Research Institute, Izatnagar, India \\ ${ }^{4}$ ICAR-Indian Veterinary Research Institute, Muktheswar, India \\ ${ }^{5}$ ICAR-CMFRI, Kochi, Kerala, India \\ *Corresponding author
}

A B S T R A C T

\begin{tabular}{|l|}
\hline Ke y w o r d s \\
Parasites, Animals, \\
Adaptation
\end{tabular}

\section{Introduction}

Parasites are organisms that derive nourishment and protection from other living organisms known as hosts. Parasites harm their hosts to foster their own needs. Most of the parasites are having a free living stage in their life cycle during which they have to survive in the absence of host and have to tolerate adverse conditions like extremes of temperature, lack of oxygen, osmotic stress, dehydration etc. On the other hand, when it is inside the host specialized habitat affords parasites a reliable source of nutrients, a relatively homeostatic environment, and protection from predators but, in exchange for these advantages, presents the special challenges of exposure to digestive enzymes, normal peristalsis and host immune response to infection. Blood and tissues seem to be harder to invade than gut as shown by the relatively small number of blood and tissue parasites in animals. Thus each parasite species must have special adaptations that guarantee infection of a host and its survival in it. Adaptation, the fitness of a parasite, results in suitable and convenient morphological and functional correlation between parasite and its environment. Adaptations possessed by the parasites can be 
morphological, nutritional, lifecycle, immunological or biochemical. A better understanding of these adaptations will help to identify weak points of parasites that will nourish our idea for designing control strategies against them.

\section{Morphological adaptations}

Size and shape: For each parasite, size and shape are unique. It depends on the environment in which they have to survive and reproduce. Many parasites are larger than their free-living counterparts. It is due to the fact that parasites usually have an unlimited food supply within the host and they have to produce a large number of offspring in order to overcome the hazards during their life cycles which necessitate infecting a host. Only few eggs or larvae manage to succeed: the larger the body, the larger the egg producing capacity. Most of the parasites are dorso-ventrally flattened owing to their need to cling on their host. Fleas and mites have flattened bodies which make them more difficult to dislodge even by scratching.

Organs of locomotion: Locomotory organs are mainly concerned with helping the parasite to reach a location where surplus food is available. But parasites habitually inhabit such places in host's body, where sufficient food is available without any effort. Thus, organs of locomotion such as cilia of turbellarians are generally absent in adult parasites except arthropods. On the other hand it is present in free living larval forms such as miracidium possess cilia as well as cercaria bears a tail for locomotion.

Organs of attachment: Despite the wide variety of parasites, there are only two trends running through the evolution of attachment organs, the development of either hooks or suckers. Suckers occur in such widely divergent groups as protozoa, monogeneans, digeneans, cestodes, parasitic crustaceans and parasitic annelids. Spines and hooks are present in many parasitic groups and the elaboration of spines or suckers or both into an eversible proboscis has occurred in the cestodes, acanthocephalans, and the acarines (ticks). Other types of attachment organ include claws in parasitic insects and the ctenidia (comb organ) of fleas. Penetrative filaments occur in a number of groups of parasite (Oxyurid nematodes, Microspora protozoans).

Organs for nutrient absorption: Nutrient acquisition is an important component of parasite pathogenesis since it is critical to parasite survival (Landfear, 2011). In endoparasites there is a trend to reduce the gut size so that they can absorb nutrients through the whole body surface. In those intestinal parasites, which do not absorb nutrients through the body surface, there is usually a thick cuticle. So helminths tend either to lose their gut or absorb nutrients through their teguments, or else retain their gut and have a thick resistant cuticle.

Nervous system: Many parasites have undergone a process referred to as sacculinization, i.e., a reduction in the complexity of sensory organs and nervous system since they have little need to detect danger in their sheltered habitat.

\section{Nutritional adaptations}

For the growth and survival of any parasite nutrition, mainly carbohydrate, protein and fat, is inevitable. Carbohydrates are essential for the parasites to generate energy, while amino acids and fatty acids are involved in the synthesis of macromolecules and the production of eggs (Dalton, 2004). Food of parasite comprises readily available and digested/ semi digested food of the host. Rather than requiring adaptations to penetrate 
the host, as ectoparasites do, endoparasites are in a nutrient-rich location so they instead have adaptations to maximise nutrient absorption. One such adaptation is the tegument, a metabolically active external cover which plays an important role in acquisition of nutrients from the host especially in the case of cestodes which is not having alimentary canal (Dalton, 2004). Here tegument is equipped with spine-like microtriches to increase the surface area that is available for nutrient acquisition (McManus, 2009). In addition to microthiches tegument is often covered with a carbohydrate-rich layer called glycocalyx which enhances nutrient absorption and secretes enzymes to aid primary digestion (Reitsma et al., 2007).

Ectoparasites have developed a series of chemosensory and behavioural adaptations that are crucial to their resource tracking capabilities on vertebrates. Olfactory receptors on antennae of insects and the first leg pair of ticks permit them to find host from a distance.

These include receptors for common respiratory products such as carbon dioxide and other breath components. Ticks, triatomine bugs and phlebotomine sandflies possess receptors for volatile products common to vertebrates such as branched short chain fatty acids and aliphatic aldehydes (Guerin et al., 2000).

Mouth parts of ectoparasites generally contain chewing mandibles, but in haematophagus insects mouthparts are modified for puncturing. In blood feeders, hypopharynx and mandibles are modified into a tube through which blood can be drawn using suction from an enlarged muscular cibarial pump. Blood feeders also usually have large salivary glands since salivary components are essential for preventing coagulation of blood while feeding.

\section{Life cycle adaptations}

Parasites usually possess an increase in reproductive potential compared with their free living relatives. Cestodes basically consist of a small head and neck region and the rest of the body consists of serially repeated gonads. Other adaptations, which increase egg production, are hermaphroditism and parthenogenesis, where every individual produces eggs and loss of seasonal reproductive cycles, so eggs and sperm are produced all the year round. Rapid maturation and extended life span also increase total reproductive capacity. Asexual reproduction which is present in different life cycle stages of the parasite is a mean to increase their reproductive potential. Parasites usually produce millions of eggs because the chance of any one egg reaching another suitable host is extremely low. In digeneans, single miracidium can give rise to hundreds of cercaria by asexual reproduction. It has been estimated that the reproductive potential of a single liver fluke (Fasciola hepatica) is four hundred million offspring in its lifetime. Yet another interesting fact is that most of the parasites are able to manipulate the behaviour of the hosts in order to increase its transmission and survival.

Parasites respond to various environmental stresses by possessing different adaptations like

Dauer larva: This term is used to describe the alternative developmental stage of nematodes such as Caenorhabditidis elegans (Cassada and Russell, 1975) and Strongyloides ratti (Viney, 1996). Under favourable environment larvae moult to $\mathrm{L}_{1}, \mathrm{~L}_{2}, \mathrm{~L}_{3}$ and $\mathrm{L}_{4}$. When the environment is unfavourable first $\left(\mathrm{L}_{1}\right)$ and second $\left(\mathrm{L}_{2}\right)$ stage larvae have the option to divert their development from reproduction to dauer formation. Signals such as temperature, food supply, dauer inducing pheromone 
(daumone) as well as daf gene (dauer formation gene) are responsible for this transformation. During dauer stage feeding is arrested indefinitely and locomotion is reduced markedly. When condition becomes favourable it will feed and will continue its development.
Diapause: It is an important survival mechanism of the parasite in temperate regions when there is absence of host. Usually exhibited by certain insects (Chapman, 1971; Romoser and Stoffolano, 1998) and nematodes like Nematodirrus battus (Evans and Perry, 1976; Wharton, 1986).

Table.1 Survival strategies of parasites inside the macrophages

\begin{tabular}{|l|l|l|l|}
\hline Sl. No & Parasite & Survival strategy & References \\
\hline 1 & Leishmania spp. & $\begin{array}{l}\text { Parasites are taken up by phagocytosis and } \\
\text { results in the formation of phagolysosome, } \\
\text { where the amastigote form can resists the } \\
\text { acidic pH and attack by lysosomal enzymes }\end{array}$ & Mauel, J. 1996 \\
\hline 2 & Trypanosoma cruzi & $\begin{array}{l}\text { Parasite can survive and divide free in the } \\
\text { cytoplasm }\end{array}$ & Hall and joiner, 1991 \\
\hline 3 & Toxoplasma gondii & $\begin{array}{l}\text { Parasite can live inside the phagosome and it } \\
\text { will resist the fusion with lysosome. ie. } \\
\text { formation of phagolysosome is inhibited }\end{array}$ & Mauel, J. 1996 \\
\hline
\end{tabular}

Hypobiosis: It is the phenomenon of temporary cessation of development of nematodes at a precise point in early parasite development and serves to synchronise the development of the parasite with events in the host and in the environment. Gastrointestinal nematodes of domestic animals are exhibiting this strategy. Although in most species fourth stage larvae is undergoing arrested development, early third stage larvae (Trichostrongylus colubriformis) and early fifth stage larvae (Dictyocaulus viviparus) can also exhibit hypobiosis. Arrested development due to host resistance is observed in the life cycle of Ostertagia ostertagi in the beginning of fourth stage larva (Smith and Grenfell, 1985)

Anhydrobiosis: In anhydrobiosis, an organism lives in a dormant, desiccated state without any requirement of water. Coiling and clumping are the two important behavioural responses shown by nematodes in this state. Coiling reduces the exposed surface area so that it can withstand drying. The infective larvae of $T$. colubriformis form tight coils when exposed to desiccation (Allan and Wharton, 1990). Here individual nematodes coil or group of nematodes aggregates in clumps to decrease cuticle surface area and this behaviour aids in slow desiccation (Solomon et al., 1999). A slow rate of desiccation will allow accumulation of trehalose, glycerol or both which act as protectants to maintain the structure of nematode membranes (Behm, 1997). Nematodes in anhydrobiosis cease all metabolic activity; they do not take up oxygen and do not significantly alter their energy reserves (Grewal, 2000). Anhydrobiosis is revived by the addition of water and the worms quickly rehydrate and return to normal locomotion in about 2 hour.

Protective structures: Physical structures such as moulted cuticle, egg shell and resistant cuticle are affording protection from extreme environment by controlling the rate of water 
loss. The third stage infective larvae of Haemonchus contortus and T. colubriformis retain the cuticle of the previous stage as a protective sheath and development is arrested until ex-sheathment occurs in the rumen of the host.

On exposed to desiccating conditions the sheath dries out first thereby reducing the rate of water loss of the enclosed third stage larvae. The lipid bilayer of the egg shell act as the permeability barrier to water loss in the case of Ascarids by which the infective stage is protected by the egg shell until ingestion by the host. Presence of strongly polar surface coat in the case of Strongyloides ratti facilitates to maintain a film of water over the cuticle by which it can tolerate desiccation.

\section{Immunological adaptations}

Despite their exquisitely developed immune system, host animals are frequently unable to rid themselves of the parasite. However, for the thousands of years that hosts and parasites have been living together and co-evolving, parasites have been searching for the right balance between hiding from and suppressing the immune system without killing the host or making it susceptible to another deadly infection. Parasites have evolved sophisticated mechanisms to evade effective host immune response and thereby enhance its survival. This include

\section{Anatomical seclusion in immunologically privileged sites}

Plasmodium lives inside the $\mathrm{RBC}$ which has no nucleus thereby it will not be recognised by cytotoxic $\mathrm{T}$ cells and natural killer cells. Toxoplasma gondi, Leishmania amastigote and Trypanosoma cruzi are residing inside the macrophage by using different survival mechanisms (Hall and joiner, 1991) (Table 1). The larval stage of the nematode Trichinella transforms the muscle cell into a specialized nurse cell that protects it and provides nutrients. This parasitic worm survives there for the lifetime of the host. Other immunologically privileged sites include the eye (Onchocerca volvulus), urinary tract (Trichomonas vaginalis) and the bile ducts (Fasciola hepatica) (Arijo et al., 1999)

\section{Antigenic variation}

The concept of immune evasion began with the demonstration of antigenic variation in African trypanosomes by Paul Ehrlich. Trypanosomes are covered by one glycoprotein (VSG) that induces immunodominant antibody response.

They are able to switch the glycoprotein regularly with the result that host mount immune response to current VSG but the parasite can switch over to another VSG which is no longer recognized by the host. Plasmodium and Giardia lamblia also use antigenic variation to trick the immune system of the host.

\section{Coating with host protein}

Tegument of trematodes and cestodes is able to absorb host components thus they can mimic as self. Schistosomes can use blood group antigens and histocompatability molecules to coat their surface.

\section{Shedding of surface antigen}

It is the main immune evasion strategy of trematodes mainly Fasciola and schistosomes

\section{Biochemical adaptations}

Parasites generally have complex life cycles, which can comprise multiple host species as well as free living stages. These complex life cycles include exposures to different 
environments, to which parasites adapt their metabolism. In some environments there might be a shortage of nutrients, forcing parasites to arrange their metabolism in such a way that metabolites are most efficiently used (Skelly et al., 1993; Boyunaga et al., 2001). It is generally accepted that free-living stages of parasitic helminths are dependent on aerobic degradation of endogenous energy sources for their energy generation since as oxygen is widely available in their habitat and these stages are small in size. They have fully active Kreb's cycle and oxidative phosphorylation. Studies conducted to explore the energy metabolism of $F$. hepatica miracidia demonstrated that it depends on aerobic degradation of their endogenous glycogen stores by glycolysis and on Krebs cycle activity for energy generation (Boyunaga et al., 2001). In contrast Schistosoma mansoni miracidia can function anaerobically also. In general the synthetic capacities of parasites are reduced when compared with their free-living relatives. Carbohydrates are degraded in the same way as occurs in mammals, glycolysis in the cytosol is followed by pyruvate decarboxylation and subsequent oxidation of acetyl-CoA by Krebs cycle in the mitochondria. Re-oxidation of the thereby formed reduced cofactors, such as $\mathrm{NADH}$, occurs by the oxygen-dependent mitochondrial electron-transport chain, which provides a proton gradient that is subsequently used for ATP synthesis. The energy metabolism of adult helminth parasites is essentially anaerobic and helminths characteristically breakdown carbohydrate into a range of organic acids such as acetate, lactate, succinate, propionate and branched chain fatty acids such as 2-methylbutyrate and 2-methylvalerate. The TCA cycle is usually reduced or modified and many parasites fix carbon dioxide and have a partial reversed cycle with phosphoenol pyruvate playing a central role. The cytochrome chain in helminths is often modified. The reduction in the TCA cycle and cytochrome chain results in a low ATP production/mole glucose catabolised. In Ascaris there is production of $6 \mathrm{ATP} / \mathrm{mole}$ of glucose, compared with 36 ATP/mole in aerobic tissues. This low ATP/mole of glucose is often compensated by high rates of glucose utilisation. There is no beta oxidation of fatty acids and limited amino acid catabolism in adult helminths (Halton, 1997). Thorough knowledge of parasite metabolism aids to exploit metabolic weak points of the parasite for development of novel drugs against them. Antibiotic therapy has not been successful for helminth infections because, unlike in the case of bacterial protozoan or viral diseases, the adult parasite itself must be removed and continued infection is not dependent upon replication of the organism.

\section{Exploiting survival strategies of parasites to control them}

Parasites have the ability to adapt and evolve to overcome the barriers put up by their hosts or the difficulties of surviving in an unpredictable environment. Now researchers are trying to identify which of the parasite's genes allow them to change their behaviour to overcome these difficult situations. This can be done by using latest biotechnological tool such as whole genome microarrays which help to compare the expression of all the genes from parasite lines that differ in their adaptations. This is particularly important while studying anthelmentic resistance wherein a single gene polymorphism renders the parasite to withstand even in the presence of drugs.

We can make use of the tricks played by the parasites to enhance its survival and transmission to design control strategies against them 
Morphologic adaptations: Since parasites have co- evolved with the host for many years, it is extremely difficult to manipulate its morphological adaptations. Still especially for external parasites attachment can be prevented by use of repellents, modified combing equipments etc.

Nutritional adaptations: Through knock-out or inhibition of crucial transporters that are highly essential for nutrient acquisition, we can control the parasites. Development of vaccine against helminth parasites by targeting digestive proteases (Shrihari et al., 2011) is an example of exploiting this adaptation.

Lifecycle adaptations: Through better management practices different developmental stages of the parasites that are exposed to the environment as well as those that are inside the intermediate hosts can be eliminated. Adequate chemotherapeutic intervention should be given to pregnant animals to prevent the maturation of hypobiotic larvae during the periparturient period. This strategy is highly essential to prevent infection to new borne animals and pasture contamination.

Immunologic adaptations: Key to the development of new drugs or vaccines is basic knowledge about how the host immune system responds to parasite infection and how the parasite thwarts the response.

Biochemical adaptations: Blocking the enzymes or receptors that are crucial for the metabolic pathways of the parasites will help to eliminate them.

Successful life cycle of a parasite depends on the extent to which they can overcome the harsh environments during its life cycle. Considering the obstacles that parasites must overcome to reproduce themselves, in finding new hosts, surviving the harsh environment, our increasing arsenal of effective antiparasiticides, it is amazing that they are able to survive and do as well as have. The morphological and biochemical reductions found in adult parasites can be considered as an economy of effort rather than a degenerate condition. Parasites have evolved behavioural adaptations to find hosts, to locate sites of infection within final and intermediate hosts, to escape immune responses, to entice another host to consume the host in which they are residing, etc. Many of these mechanisms are highly complex, but they are sometimes also quite subtle. While the mechanisms or processes involved in a few parasites are understood, most are not. Thorough analysis of these mechanisms is the need of the hour to understand the weak points of these parasites. This will help us to design a better control strategy as well as to modify the existing control measures against them.

\section{References}

Allan, G.S. and Wharton, D.A. (1990). Anhydrobiosis in the infective juveniles of Trichostrongylus colubriformis (Nematoda: Trichostrongylidae). Int. J. Parasitol. 20: 183-92.

Arijo, A. G., Anwar, A. H. and Soomro, N. M. (1999). Evasion of host immune response in parasites: A review. Pakistan Vet. J. 191: 1-3.

Behm, C.A. (1997). The role of trehalose in the physiology of nematodes. Int. J. Parasitol. 27: 215-229.

Boyunaga, H., Schmitz, M. G., Brouwers, J. F., Van Hellemond, J. J. and Tielens, A. G. (2001). Fasciola hepatica miracidia are dependent on respiration and endogenous glycogen degradation for their energy generation. Parasitology. 122: 169-173.

Cassada, R. and Russell, R. (1975). The Dauerlarva: A post-embryonic developmental variant of the nematode $C$. elegans. Dev. Biol. 46: 326-342. 
Chapman, R. F. (1971). The insects. Structure and function. American Elsevier Publishing Company, New York.

Dalton J. P. (2004). Role of the tegument and gut in nutrient uptake by parasitic platyhelminths. Canadian J. Zool. 82: 211-232.

Evans, A. A. F. and Perry, R. N. (1976). Survival strategies in nematodes. The organization of nematodes. Academic Press, London pp. 383-424.

Guerin, P. M., Krober, T., McMahon,C., Guerenstein, P., Grenacher, S., Vlimant, M., Diehl, P., Steuliet, P. and Syed, Z. (2000). Chemosensory and behavioural adaptations of ectoparasitic arthropods. Nova Acta Leopoldina. 83: 213-229.

Grewal, P.S. (2000). Enhanced ambient storage stability of an entomopathogenic nematode through anhydrobiosis. Pest Management Science 56: 401-406.

Hall, F. and Joiner, K. A. (1991). Strategies of obligate intracellular parasites for evading host defence. Immunoparasitol. Today. 7: A22- A27.

Halton, D. W. (1997). Nutritional adaptations to parasitism within the platyhelminthes. Int J Parasitol. 27: 693-704.

Landfear, S.M. (2011). Nutrient transport and pathogenesis in selected parasitic protozoa. Eukaryot. Cell. 10: 483-493.

Mauel, J. (1996) Intracellular survival of protozoan parasites with special reference to Leishmania spp, Toxoplasma gondii and Trypanosoma cruzi. Adv. Parasitol. 38: $1-51$

McManus, D.P. (2009). Reflections on the biochemistry of Echinococcus: past, present and future. Parasitology. 136: 1643-1652.

Reitsma, S., Slaaf, D. W., Vink, H., van Zandvoort, M. A. and Egbrink, M. G. (2007). The endothelial glycocalyx: composition, functions, and visualization. European J. Physiol. 454: 345-359.

Romoser, W. S. and Stoffolano, J. G. (1998). The Science of Entomology, 4th ed. WCB/McGraw-Hill, Boston, MA.

Shrihari, N., Mariraj, J., Kumudini, T.S. and Krishna S. (2011). Intestinal perforation due to Tapeworm: Taenia Solium. J. Clin. Diagn. Res. 5: 1101-1103.

Skelly, P. J., Stein, L. D. and Shoemaker, C. B. (1993). Expression of Schistosoma mansoni genes involved in anaerobic and oxidative glucose metabolism during the cercarial to adult transformation. Mol. Biochem. Parasitol. 60: 93-104.

Smith, G. and Grenfell, B. T. (1985). The population biology of Ostertagia ostertagi. Parasitol. Today. 1: 76-81.

Solomon, A., Paperna, I. and Glazer. I. (1999). Desiccation survival of the entomopathogenic nematode Steinernemat feltiae: induction of anhydrobiosis. Nematology. 1: 61-68.

Viney, M. E. (1996). Developmental switching in the parasitic nematode Strongyloides ratti. Proc. Biol. Sci. 263: 201-208.

Wharton, D. A. (1986). A functional biology of nematodes. The Johns Hopkins University Press, Baltimore, MD.

\section{How to cite this article:}

Siju Susan Jacob, P.P. Sengupta, J.K. Chamuah, O.K. Raina, M. Sankar, T.G. Sumithra and Prajapati, A. 2018. Exploitation of Adaptations of Animal Parasites - A Key towards Designing Effective Control Strategies against Parasitic Diseases of Animals. Int.J.Curr.Microbiol.App.Sci. 7(05): 3355-3362. doi: https://doi.org/10.20546/ijcmas.2018.705.392 